\title{
Exploring Patient Reactions to Pen-Tablet Computers: A Report from CaReNet
}

\author{
Deborab S. Main, $P b D$ \\ Javan Quintela, BS \\ Rodrigo Araya-Guerra, BA \\ Sherry Holcomb \\ Wilson D. Pace, MD \\ Department of Family Medicine, University \\ of Colorado Health Sciences Center, Aurora, \\ Colo
}

\begin{abstract}
PURPOSE We wanted to study patient receptivity to using pen-tablet computers for collecting data in a practice-based research network.

METHODS We analyzed exit interviews and field notes collected by trained research assistants as part of a larger Colorado Research Network (CaReNet) study comparing pen-tablet and paper-pencil methods to collect data for the Primary Care Network Survey (PRINS).

RESULTS A total of 168 patients completed a patient exit interview after completion of the pen-tablet-based survey instrument. Analyses of these brief interviews and field notes indicated that patients had favorable reactions to using pen-tablet computers. The most common barriers were related to glitches in the technology; the voice recognition software was the most problematic, with patients (as well as clinicians) finding this feature to be frustrating.
\end{abstract}

CONCLUSIONS Patients were able and willing to use pen-tablet computers for completing forms within busy primary care offices. Increasing patient involvement in practice-based research may be even more practicable through the use of this novel technology, which can allow patient-directed data collection at a single point in time as well as longitudinally.

Ann Fam Med 2004;2:421-424. DOI: 10.1370/afm.92

\section{INTRODUCTION}

s we increase the number of practice-based research networks
(PBRNs) and their capacity to address important health issues
facing primary care practices and their patients, we need to improve the quality and feasibility of research in these practice settings. Such improvements stem from the need to collect better data to describe primary care processes and outcomes and to develop more useful, efficient methods that do not disrupt the flow of busy primary care offices. In practice-based research, issues of time; disruption of the doctor-patient relationship ${ }_{i}$ and clinician, office staff, and patient receptivity are high on the wish list for improving our data collection methods.

Although previous studies have shown the value of computers and touch screen technology as clinical tools for patient use, ${ }^{1-3}$ the use of these devices in practice-based research has received far less attention. In fact, most primary care research to date has focused on the feasibility and receptivity of new forms of technology from the clinician's perspective, ${ }^{4,5}$ with few studies specifically addressing how patients feel about participating in computer-directed research. As part of a larger study exploring the utility of using computers in practice-based research networks (AHRQ P20 HS11228-01), we examined the qualitative results of patient exit interviews and field notes on patient receptivity to using pen-tablet computers for completing a brief, self-report questionnaire. 


\section{METHODS}

\section{Study Sample}

For the present study, our subjects were patients of clinicians from 11 urban primary care practices in the Colorado Research Network (CaReNet) who agreed to participate in the Primary Care Network Survey (PRINS). Participating sites were 2 community health centers that serve a large percentage of rural disadvantaged populations, 4 residency practices that serve a large percentage of urban disadvantaged patients, 3 university-affiliated practices, and 2 urban private practices.

\section{Patient Recruitment}

CaReNet included a patient component to our PRINS study to describe our patient population and to determine the feasibility of using pen-tablets as a data collection method. A research assistant approached patients about participating in the study as they checked in for their clinic appointments. The study was explained in detail, and the research assistant reviewed the consent form with the patient and obtained his or her signature. After consenting to the study, patients were randomized to a paper or an electronic questionnaire study group. Those assigned to using the pen-tablet were taught how to use the new computer technology and a voice recorder, which was used to collect information on reasons for visit. Because we obtained consent from the patients before they were assigned to paper or pentablet study group, we could not calculate differences in response rates for each data collection method.

\section{Patient Survey}

As a result of our desire to know more from our patients' perspectives, CaReNet developed a patient survey instrument using a subset of questions from the PRINS clinician survey (sex, race-ethnicity, insurance status, reason for visit) and additional questions from other standardized questionnaires. These additional questions elicited information about the patients' living environment, family, and ability to pay, ie, information that was not usually available to clinicians but that might provide valuable insight when providing care.

\section{Pen-Tablet Data Collection}

We programmed Fujitsu PenCentra 200 pen-tablet computers using the Microsoft Windows for Handheld CE 2000 operating system. The survey instrument was programmed using Windows CE Visual Basic Version 3.0. Because reason for visit required free-text data entry, patients were offered a voice-recording option for which they had to open a new window and use the CE interface for this function. After patients had completed their questionnaire, they were asked to deliver the pen-tablet computer to their clinicians for their completion of the clinician portion of PRINS. In most cases, clinicians were asked to return the computer to the patient so the patient could return the computer to the front desk or the research assistant. In high-volume practices, clinicians returned the tablet to the research assistant so that patient flow was not disrupted.

\section{Study Feedback Exit Interview}

The research assistant asked patients randomized to both the paper arm and the electronic arm of the study to participate in a 5 -question exit interview about their experience. This interview took no more than 1 to 2 minutes and was conducted after the patients had completed their questionnaires and had been seen by their clinicians. The patient questionnaire, the patient study feedback interview, and the patient consent forms were available in English and Spanish, and the research assistants spoke both English and Spanish.

For the purposes of this article, we focus primarily on data related to patient use of pen-tablets.

\section{Data Analysis}

Using both site visit field notes and 168 patient exit interviews, we were able to learn more about patient receptivity to pen-tablet based data collection. These qualitative data were entered into an Access database and coded using ATLAS.ti (Scientific Software Development, Berlin, Ger) to explore patient reactions and responsiveness to pen-tablets. Although these data involved only "thin descriptions" of how patients felt, qualitative software helped us organize data across practices and patients and conduct simple content analyses of patient-reported difficulty and reactions to pen-tablets, including reported barriers. Data were content coded and summarized by 1 author (DSM), then verified and expanded by 2 research assistants (JQ, RA) who implemented the study in CaReNet practices.

\section{RESULTS}

Overall, patients were able and willing both to complete pen-tablet-administered questionnaires and to deliver pen-tablet computers to clinicians and back to research assistants. Research assistants reported that it took anywhere from 2 to 4 minutes to train patients to use pen-tablets; at least one half of this time was devoted to showing patients how to use the voice-recording device. Most patients seemed comfortable with the pen-tablet technology. In fact, the small size and ease of use of the pen-tablet were appealing for many participating patients. When asked in exit interviews whether the pentablet was difficult to use, more than $70 \%$ of patients in this condition indicated that it was not. The following are typical patient responses when asked whether the 
pen-tablet was difficult: "No, everything was easy." "No, I am a computer user." "No, I'm computer liberate, so I was able to plow right through it." Research assistants reported the following: "Patient reactions to participating in study varied, but most welcomed the study." "Some patients seemed reluctant, but upon commencing survey they completed it quickly." "Some patients need time for the learning curve in computers/tablets."

Of the $30 \%$ of patients who reported any difficulty, by far the greatest number of pen-tablet users ( $\mathrm{n}=$ 26) reported difficulty recording their reason for visit using the voice-recording technology. Patient-reported barriers included confusion about the correct steps in activating and stopping the voice recorder, discomfort about verbalizing their reasons for visit in the waiting room among others, discomfort with sharing this information at all, and glitches in the voice activation software that caused the computer to freeze or shut down: "No [problem with completing the survey], skipped over recording question because it freezes." No, a breeze! Except for the recording question." "The recording question was difficult and confusing." "Recording part was difficult; feels like the survey is interrupted." "Recording was hard to figure out."

Research assistant field notes reinforced exit interview findings. One particular finding was that patients filling out the electronic version had difficulty recording while they were reading the instructions: "Patients are having difficulty with the question that asks them to record their response. Some patients and clinicians call on RAs to facilitate this portion of the survey. Patients have indicated that the recording instructions are not helpful because they disappear once they turn on the recording application."

As indicated in the above quote, several participants (more than 12) who experienced difficulty (caused by difficulty with voice recording and more general discomfort with computers) requested and received assistance from the research assistants. Research assistants reported that most of these patients were elderly. A few pen-tablet users commented about the size of the font (too small), the glare on the computer screen, or the small size of the buttons: "Kind of hard to read the screen." "Recording was confusing, buttons too small." "Print should be bigger, even frame could be bigger; glare is bothersome."

One research assistant noted: "Patients need help with the recording question, it seems that the buttons are not as big as they should be. The sunshine affects the visual efficacy of the tablet's screen."

Despite being assigned to the pen-tablet survey group, a small percentage of patients $(4,2.5 \%)$ elected to change to paper once the pen-tablet was introduced; nearly all were elderly.
The research assistant indicated, "We have had several participating patients who have been randomized to participate using the tablet elect to complete the paper version of the questionnaire during the course of our data collection here, even though we have introduced them to the tablet. They ask if they could participate on condition that they do not have to use the tablet."

\section{DISCUSSION}

Patients were generally receptive and comfortable completing the questionnaire using pen-tablet technology. Importantly, training patients on pen-tablet computer use took minimal time. The pen-tablet was clearly labeled to make it easy for patients to enter their responses by pushing buttons. Indeed, exit interviews and field notes indicated that patients expressed little frustration with entering data into the tablet. This finding is consistent with the findings of a recent study ${ }^{6}$ that looked at prenatal patient reactions to using similar touch screen technology. They found that, even among poor women with relatively little experience with touch screen computers, a large percentage (96\%) reported not having any difficulty with the technology.

In the present study, however, we added one complicating feature to our pen-tablet data collection: automated voice recording. This additional step caused considerable difficulty for many pen-tablet users. Patients indicated that the recording device was confusing to use, it froze the computer, or they were not sure whether they had operated it correctly. It is important to note that study clinicians also had similar difficulties in recording patient reasons for visit using the pen-tablet recorder; in fact, many elected to write (rather than record) their responses, or they used the touch screen graffiti-recognition feature not offered to patients. Importantly, despite these computer and voice recorder glitches, patients viewed pen-tablets quite positively, particularly as a method for completing closed-ended questionnaires.

As with any new technology, many of these challenges are expected and often disappear with newer models and software. Since the present study was undertaken, improved pen-tablet computers have become available. These computers operate a full version of Windows XP, offering more advanced programming languages, larger and improved screens, and full integration of all functions. The patient concerns about screen size, font size, voice-recording controls, and button size can all be addressed with current PC pen-tablet devices.

In the present study, patients were both participants and collaborators in the data collection process. Once patients completed their questionnaire, they carried the computer to their clinician, waited for them to complete 
their portion, and then returned it to the front desk or research assistant. Patients seemed quite comfortable with this role, with the greatest challenge involving the time constraints placed on the medical visit. Patientdirected data collection holds considerable promise both as an effective method for data collection and for activating both patients and clinicians during the medical visit. In addition, this method offers promise for future research involving patients in longitudinal data collection within PBRNs. This idea is certainly not new. In fact, one study nearly a decade ago successfully involved rheumatoid arthritis patients in daily data collection to determine their response to treatment. ${ }^{7}$ They found that patient-directed data were more sensitive than data collected through standard assessments done in weekly clinic visits, even though patients used less sophisticated computer technology for data entry.

There are several limitations that must be considered while interpreting the qualitative results of this study. First, because we asked and received consent from patients before assignment to pen-tablet or paper data collection groups, we were unable to determine precise differential response rates by type of collection method-an important indicator of patient receptivity to this technology. We do, however, have data suggesting that the use of pen-tablets did not decrease and may even have enhanced patient response rates. We found that $67 \%$ of the patients in the randomized paper or pen-tablet groups agreed to participate, compared with $49 \%$ of patients in the paper-only group, suggesting that patients did not seem deterred by the possibility that they would be using pen-tablet computers.

Second, patients participating in this qualitative study might not represent the general patient population of CaReNet practices, or other primary care practices for that matter. This qualitative study included only the subset of patients randomly assigned to using the pen-tablet, and these study patients were assigned to the pen-tablet data collection method only if their clinician agreed to participate in both forms of data collection. In reality, only 35 of 67 participating clinicians participated in the randomized portion of this study, thus introducing a potential bias in those patients participating in this study. Certainly, understanding clinician reaction to pen-tablet technology is another important future study.

Third, the present study does not examine whether there are meaningful differences in the content or quality of the data collected using both methods. Several studies, however, including a recent study of a quality-of-life instrument ${ }_{1}^{8}$ have examined this issue in a range of patient populations and settings and found few meaningful differences between the quality of data collected by paper and pencil and that collected by computers. Finally, although these qualitative data were useful for identifying general patient reactions to this newer form of technology, their nature precluded us from offering greater insight or in-depth analysis of patient experiences with the patient-computer or patient-computer-clinician interface-an important area for future research.

In this qualitative study, we found that patients are generally comfortable using handheld computers and require little training on their use. This novel, easy-touse, technology can enhance patient involvement in both clinical care and the research process. Patients can play a coordinating role in data collection by delivering these devices to their clinicians during visits and returning them to office staff afterward. Pen-tablet technology used by both the patient and the clinician offers potential benefits for both practice-based research and clinical activities within ambulatory care.

To read or post commentaries in response to this article, see it online at http://www.annfammed.org/cgi/content/full/2/5/421.

Key words: Research design, practice-based; patients; computers; data collection; surveys

Submitted May 13 2003; submitted, revised, August 19, 2003; accepted September 7, 2003.

\section{References}

1. Porter SC, Silvia MT, Fleisher GR, Kohane IS, Homer CJ, Mandl KD. Patients as direct contributors to the medical record: validation of their electronic input. Ann Emerg Med. 2000;35:346-352.

2. Bock B, Niaura R, Fontes A, Bock F. Acceptability of computer assessments among ethnically diverse, low-income smokers. Am J Health Prom. 1999;13:299-304.

3. Bachman JW. The patient-computer interview: a neglected tool that can aid the clinician. Mayo Clin Proc. 2003;78:67-78.

4. Criswell DF, Parchman ML. Handheld computer use in US family practice residency programs. J Am Med Inform Assoc. 2002;9:80-86.

5. Hart-Hester S, Olvtade J, Arthur CR. Utilization of computer technology by family medicine community preceptors in Mississippi. J Miss State Med Assoc. 2002;43:273-276.

6. Thornberry J, Bhaskar B, Krulewitch CJ, et al. Audio computerized self-report interview use in prenatal clinics. Comput Inform Nurs. 2002;20:46-52

7. Nived O, Sturfelt SA, Singer P. A comparison of 6 months' compliance of patients with rheumatoid arthritis treated with tenoxicam and naproxen: use of patient computer data to assess response to treatment. J Rheumatol. 1994;21:1537-1541.

8. Kleinman L, Leidy NK, Crawley J, Nonomi A, Schoenfeld P. A comparative trial of paper-and-pencil versus computer administration of the quality of life in reflux and dyspepsia (QOLRAD) questionnaire. Med Care. 2001;39:181-189. 